\title{
Comparison of library preparation methods reveals their impact on interpretation of metatranscriptomic data
}

Adriana Alberti ${ }^{1 *+}$, Caroline Belser ${ }^{1 \dagger}$, Stéfan Engelen ${ }^{1}$, Laurie Bertrand ${ }^{1}$, Céline Orvain ${ }^{1}$, Laura Brinas ${ }^{1}$, Corinne Cruaud ${ }^{1}$, Laurène Giraut ${ }^{1}$, Corinne Da Silva' ${ }^{1}$, Cyril Firmo ${ }^{1}$, Jean-Marc Aury ${ }^{1}$ and Patrick Wincker ${ }^{1,2,3^{*}}$

\begin{abstract}
Background: Metatranscriptomics is rapidly expanding our knowledge of gene expression patterns and pathway dynamics in natural microbial communities. However, to cope with the challenges of environmental sampling, various rRNA removal and CDNA synthesis methods have been applied in published microbial metatranscriptomic studies, making comparisons arduous. Whereas efficiency and biases introduced by rRNA removal methods have been relatively well explored, the impact of cDNA synthesis and library preparation on transcript abundance remains poorly characterized. The evaluation of potential biases introduced at this step is challenging for metatranscriptomic samples, where data analyses are complex, for example because of the lack of reference genomes.
\end{abstract}

Results: Herein, we tested four cDNA synthesis and Illumina library preparation protocols on a simplified mixture of total RNA extracted from four bacterial species. In parallel, RNA from each microbe was tested individually. cDNA synthesis was performed on rRNA depleted samples using the TruSeq Stranded Total RNA Library Preparation, the SMARTer Stranded RNA-Seq, or the Ovation RNA-Seq V2 System. A fourth experiment was made directly from total RNA using the Encore Complete Prokaryotic RNA-Seq. The obtained sequencing data were analyzed for: library complexity and reproducibility; rRNA removal efficiency and bias; the number of genes detected; coverage uniformity; and the impact of protocols on expression biases. Significant variations, especially in organism representation and gene expression patterns, were observed among the four methods. TruSeq generally performed best, but is limited by its requirement of hundreds of nanograms of total RNA. The SMARTer method appears the best solution for smaller amounts of input RNA. For very low amounts of RNA, the Ovation System provides the only option; however, the observed biases emphasized its limitations for quantitative analyses.

Conclusions: cDNA and library preparation methods may affect the outcome and interpretation of metatranscriptomic data. The most appropriate method should be chosen based on the available quantity of input RNA and the quantitative or non-quantitative objectives of the study. When low amounts of RNA are available, as in most metatranscriptomic studies, the SMARTer method seems to be the best compromise to obtain reliable results. This study emphasized the difficulty in comparing metatranscriptomic studies performed using different methods.

Keywords: Metatranscriptomics, cDNA synthesis method, Gene expression

\footnotetext{
*Correspondence: aalberti@genoscope.cns.fr; pwincker@genoscope.cns.fr

${ }^{\dagger}$ Equal contributors

${ }^{1}$ CEA-Institut de Génomique, Genoscope, Centre National de Séquençage, 2

rue Gaston Crémieux, CP5706, F-91057 Evry Cedex, France

2Université d'Evry, UMR 8030, CP5706 Evry, France

Full list of author information is available at the end of the article
} 


\section{Background}

Metatranscriptomic approaches based on next generation sequencing have emerged as a powerful tool to provide insight into microbial activity in complex environmental communities, such as marine [1-7], soil [8-10] or human internal organ ecosystems [11]. However, these studies have used many different methods for cDNA synthesis, making comparison of the results difficult and highlighting the challenges of analyzing RNA samples from environmental communities. Indeed, due to sampling constraints, the quality and quantity of RNA available for library preparation may be very heterogeneous and limited $[12,13]$. For these reasons, the most common strategies used for cDNA synthesis rely mainly on polyadenylation of prokaryotic mRNA, followed by a linear or exponential amplification of cDNA $[1-7,11]$. Usually, an rRNA removal treatment precedes the cDNA synthesis, to reduce the predominant rRNA fraction (which can be more than 95\%) [14]. Different methods for ribosomal RNA removal have been reported [15-17] and compared in GC-rich transcriptomes [18], and in synthetic [19] and natural microbial communities [20,21], giving an extensive overview on their efficiency and biases. By contrast, even if most studies validate the transcript abundance inferred by sequencing data using quantitative real-time reverse transcription PCR (qRT-PCR) to check for artefacts in cDNA amplification [1-4], the bias introduced at this step remains poorly explored.

Recently, several new cDNA preparation methods especially conceived for RNA-Seq have been released and, for some of them, the impact on data analysis and interpretation in eukaryotic transcriptomes has been reported [22-24]. Our work aimed to expand upon these studies by focusing on microbial metatranscriptomics. We tested three methods that should, in principle, permit the experiment to be initiated from small inputs, equal to or lower than $100 \mathrm{ng}$ total RNA, a realistic quantity available from environmental sampling. We prepared libraries with SMARTer Stranded RNA-Seq Kit from Clontech (SMART), the Ovation RNA-Seq System V2 (OV) and the Encore Complete Prokaryotic RNA-Seq System (ENC, recently renamed Ovation Complete Prokaryotic RNA-Seq) from NuGEN and compared them with the TruSeq Stranded Total RNA Library Preparation from Illumina (TS), one of the most widely applied methods for RNA-Seq studies (for a methods overview, see Table 1). Whereas TS and SMART cDNA protocols are based on synthesis by random priming after RNA fragmentation, the ENC synthesis is carried out using selective primers with decreased affinity for rRNA sequences, with no need for prior ribosomal depletion. Finally, the OV system allows cDNA synthesis by oligo $(\mathrm{dT})$ and random priming. Even if this protocol is not especially developed for prokaryotic RNA-Seq, it proved to be very efficient to detect viral RNA sequences in clinical samples containing ultra-low amounts (some femtograms) of viral RNA genomes [25]. Therefore, we chose to test it because of its robustness in library yield when very low amounts of RNA are available. This is possible because of a linear amplification step that allows the production of micrograms of double-stranded cDNA from a few nanograms of RNA.

In this study, we tested the four RNA-Seq methods on four well-known microbial species, individually and pooled together, and assessed their performances. We evaluated rRNA depletion efficiency and bias; library complexity; alignment efficiency; gene detection sensitivity and abundance; evenness of transcript coverage; and technical reproducibility. Our results revealed the strengths and weaknesses of each method, and provided guidelines for choosing the method that is best adapted for metatranscriptomic studies.

\section{Results}

\section{Experimental design}

To overcome the extreme complexity of environmental samples, we set up a simplified benchmark comprising total RNA from four microbial species: two Gram negative organisms, Escherichia coli MG1655 and Acinetobacter baylyi ADP1; and two Gram positive organisms, Lactococcus lactis MG1363 and Bacillus subtilis 168. The four species, for which reference genomes are available, have different GC contents, heterogeneous genome sizes and gene contents (Additional file 1: Table S1). They were cultivated in different conditions reflecting their different gene expression dynamics.

We prepared libraries using total RNA (control libraries) and depleted RNA individually from the four microorganisms and from a pool of four RNAs (hereafter named MIX) in duplicate, starting from the same individual RNAs and MIX sample. Using Illumina paired-end sequencing, we generated, on average, 20.5 M reads and $0.8 \mathrm{M}$ reads for the control and depleted RNA libraries, respectively (Table 2 ). Reads were cleaned as described in the Methods and subsequent analyses were performed on the remaining data (cleaned reads). Here we discuss the data analyses of the L. lactis and MIX datasets. The results obtained for the other bacterial species are mostly presented in the Additional files.

\section{rRNA removal efficiency}

rRNA depletion using the Ribo-Zero kit was efficient: the proportion of rRNA reads was less than $1 \%$ for the TS and OV libraries, and less than 3\% for the SMART library (Table 2). By contrast, this proportion increased to $38 \%$ in the ENC libraries, suggesting that the selective priming in the ENC method is not efficient. This led to a decreased number of useful reads, which could negatively affect 
Table 1 Summary of the principal characteristics of the RNA-Seq library preparation kits evaluated in this study

\begin{tabular}{|c|c|c|c|c|}
\hline & TruSeq stranded & Encore complete & Ovation RNA-Seq V2 & SMARTer stranded \\
\hline $\begin{array}{c}\text { RNA input } \\
\text { (minimal requirement according } \\
\text { to the manufacturer) }\end{array}$ & 100 ng depleted RNA & $100 \mathrm{ng}$ total RNA & 0,5 ng depleted RNA & 1 ng depleted RNA \\
\hline Minimum quality & FFPE & $\mathrm{RIN}>7$ & FFPE & FFPE \\
\hline rRNA depletion required & Yes & No & Yes & Yes \\
\hline cDNA synthesis & Random primers & Selective priming & Random and oligo(dT) primers & Random primers \\
\hline Fragmentation method & RNA by divalent cations + heat & cDNA by Covaris shearing & cDNA by Covaris shearing & RNA by heat \\
\hline Strand selection & Yes & Yes & No & Yes \\
\hline $\begin{array}{l}\text { Library preparation method } \\
\text { and reagents }\end{array}$ & Included & Included & Not included & Included \\
\hline Multiplex capacity & 96-plex & 16-plex & $\begin{array}{l}\text { according to the library } \\
\text { preparation method chosen }\end{array}$ & 12-plex \\
\hline \multirow[t]{2}{*}{ Experiment duration } & 6 hours & 7 hours & 4.5 hours for cDNA synthesis & 4.5 hours \\
\hline & & & + time for library preparation & \\
\hline
\end{tabular}

FFPE: Formalin-fixed, paraffin-embedded tissue.

RIN: RNA integrity number.

metatranscriptomic studies where deep sequencing is needed to obtain sufficient coverage.

\section{Library complexity}

We assessed the complexity of each library by calculating the number of duplicated paired reads. We preferred to evaluate the duplication on paired reads rather than on single reads, as they take into account the PCR duplicates. The results (Table 2) showed satisfactory values for all experiments, except for the SMART control libraries. The very high duplication rate in these libraries could reflect an insufficient amount of input RNA

Table 2 Sequences and mapping statistics

\begin{tabular}{|c|c|c|c|c|c|c|c|c|c|c|}
\hline \multirow{3}{*}{ Library name } & \multicolumn{2}{|c|}{ Raw reads (millions) } & \multicolumn{2}{|c|}{$\%$ rRNA $^{a}$} & \multicolumn{2}{|c|}{ Cleaned reads $^{\mathbf{b}}$ (millions) } & \multicolumn{2}{|c|}{$\%$ mapped reads ${ }^{c}$} & \multicolumn{2}{|c|}{$\%$ duplication rates ${ }^{d}$} \\
\hline & replicate & replicate & replicate & replicate & replicate & replicate & replicate & replicate & replicate & replicate \\
\hline & 1 & 2 & 1 & 2 & 1 & 2 & 1 & 2 & 1 & 2 \\
\hline TS_L.L & 0.724 & 0.747 & 0.14 & 0.23 & 0.720 & 0.743 & 77.2 & 72.3 & 2.19 & 5.84 \\
\hline ENC_L.L & 0.764 & 0.739 & 36.6 & 37.96 & 0.481 & 0.454 & 67.1 & 51.2 & 5.52 & 13 \\
\hline OV_L.L & 0.809 & 0.792 & 0.2 & 0.39 & 0.802 & 0.784 & 65.3 & 61 & 2.3 & 2.45 \\
\hline SMART_L.L & 0.904 & 0.697 & 2.9 & 2.88 & 0.837 & 0.650 & 62.9 & 63.7 & 8.76 & 7.62 \\
\hline TS_L.L control & 22.161 & 19.845 & 95.6 & 95.4 & 0.960 & 0.885 & 71.2 & 71.4 & 8.47 & 6.92 \\
\hline OV_L.L control & 21.989 & 17.513 & 71 & $78 ., 5$ & 6.302 & 3.676 & 73 & 74.4 & 1.01 & 0.57 \\
\hline SMART_L.L control & 24.806 & 18.798 & 95 & 95.1 & 1.146 & 0.831 & 51.5 & 51.9 & 54 & 61.6 \\
\hline TS_MIX & 0.754 & 0.769 & 0.21 & 2.27 & 0.748 & 0.747 & 73.1 & 72.1 & 1.23 & 1.59 \\
\hline ENC_MIX & 0.749 & 0.732 & 37 & 36.61 & 0.470 & 0.461 & 66.5 & 66 & 7.4 & 5.58 \\
\hline OV_MIX & 0.864 & 1.177 & 0.9 & 0.6 & 0.849 & 1.161 & 55.1 & 54.7 & 0.93 & 0.85 \\
\hline SMART_MIX & 0.824 & 0.726 & 0.77 & 0.96 & 0.809 & 0.702 & 65.7 & 66.9 & 6.5 & 7.03 \\
\hline TS_MIX control & 20.257 & 30.693 & 93 & 94.1 & 1.393 & 1.775 & 70.4 & 71.1 & 3.24 & $3 ., 28$ \\
\hline OV_MIX control & 19.510 & 17.984 & 84 & 82 & 2.983 & 3.215 & 75.2 & 70.4 & 1.78 & 0.39 \\
\hline SMART_MIX control & 14.699 & 18.603 & 93.5 & 93.5 & 0.915 & 1.160 & 63.9 & 61.9 & 23 & 39 \\
\hline
\end{tabular}

L.L: library prepared from L. lactis depleted RNA.

L.L control: library prepared from L. lactis total RNA.

_MIX: library prepared from the MIX depleted RNA.

MIX control: library prepared from the MIX total RNA.

a proportion of rRNA reads detected in the raw reads.

${ }^{b}$ number of sequences remaining after the data quality control pipeline treatment applied on raw reads.

cproportion of cleaned reads uniquely mapped on CDS sequences.

${ }^{d}$ duplication rate estimated on 100000 cleaned reads. 
(1 ng), which resulted in a low final library yield. Consequently, the number of useful reads in these libraries was lower. The duplication rates in the OV and ENC libraries were not comparable to the others, because in these preparations, cDNAs are randomly sheared after their synthesis. Indeed, this fragmentation step introduces additional diversity into the starting position of the sequence.

\section{Transcript coverage and orientation}

Reads from the L. lactis libraries were aligned against the L. lactis CDS sequences to estimate the specificity of each method. TS produced the best mapping percentages to the CDS sequences (72 and 77\%), followed by OV and SMART (61 to 65\%) (Table 2). Mapping rates were variable for the ENC samples (51 and 67\%), indicating that this protocol was less reproducible.

Based on L. lactis gene prediction, we assessed the orientation of the reads in SMART, ENC and TS libraries, which were expected to preserve the coding strand information. Around 99\% of SMART and ENC reads mapped to the sense strand, whereas around $99 \%$ of TS reads mapped to the antisense strand, as expected according to the manufacturer's specifications. These strand-specific methods are advantageous for de novo transcriptome assembly, gene annotation and detection of potential antisense transcription.

The ENC protocol had the highest rate of intergenic covered bases (21\%) and OV had the lowest (13\%) (Additional file 1: Table S2). As a rigorous DNAse treatment was applied, we could infer that the presence of reads mapping to intergenic regions reflected the capture of RNAs at different steps of their maturation or synthesis. Indeed, the same RNA extractions were used for each experiment, suggesting that these differences in the percentages of intergenic regions could be attributed to the different RNA reverse transcription protocols.

We evaluated the evenness of CDS coverage for each method, based on the L. lactis CDS coverage extracted from the MIX experiments. TS, OV and SMART covered the CDS globally over their entire lengths, with a drop at the $5^{\prime}$ and $3^{\prime}$ extremities. ENC had a higher coverage rate at the $5^{\prime}$ extremities. Plots of CDS-accumulated coverage versus their $\mathrm{GC}$ content showed that high $\mathrm{GC}$ content CDS were detected less frequently in OV libraries, whereas the other methods presented the same global profile for all the CDS, irrespective of GC content (Figure 1).

\section{Metatranscriptome bias evaluation}

The MIX samples were especially useful for assessing if the cDNA and library preparation methods had introduced bias into the pattern of gene expression from each bacterial species in a multispecies context. After alignment of MIX library reads against CDSs, we observed that the distribution of reads attributed to each organism was similar for the TS, SMART and ENC samples (Additional file 1: Table S3). However, the distribution of the OV reads was quite different: in particular, $38 \%$ of the reads were attributed to L. lactis, compared with 20-25\% for the other methods. Again, the OV method seemed to introduce a bias in relation to the GC content of the genomes (Additional file 1: Table S1).

We then normalized the RNA-Seq fragment counts by calculating the fragments per kilobase of CDS per million fragments mapped (FPKM) and performed a linear correlation between the L. lactis and MIX samples. L. lactis mRNA abundances correlated well in the MIX and the single species samples for all the methods, except for SMART (Table 3 and Additional file 2: Figure S1). For this method, however, almost all the CDSs in the L. lactis sample were present in the MIX. Indeed, we observed that absent genes in the MIX had the lowest FPKM values in the L. lactis sample. The L. lactis RNA quantity used in the MIX sample preparations was four-fold less than that in the L. lactis libraries. We inferred that this led to a loss of information, essentially for the lowest-expressed genes.

\section{Experimental reproducibility}

To test the robustness of each method, the Pearson correlation coefficients between the two replicates of the L. lactis samples were calculated (Table 3). The OV and SMART methods performed best (Pearson correlation coefficient $r=0.995)$, whereas the ENC method was the least reproducible $(\mathrm{r}=0.736)$ (Table 3 and Additional file 2: Figure S2). The variability in the CDS detection between two replicates was around 8\%, except for ENC (11\%). For the MIX samples, replicates for each of the four methods all correlated well (Additional file 2: Figure S3).

\section{Gene expression level}

We compared the gene expression patterns to examine how they were affected by the different cDNA synthesis methods. We considered the TS experiments as a reference and calculated the Pearson correlation and the percentage of differentially expressed genes (DEGs) for each method in the L. lactis samples (Tables 3 and 4, Additional file 2: Figures S4 and S5). This choice was based on the fact that TruSeq experiments are not made with limited amounts of RNA and should better reflect the complexity of the transcriptome. Indeed, in this study, experiments performed with TS produced the best results in terms of library complexity, gene detection and coverage, and reproducibility.

Surprisingly, broad differences in gene expression patterns were observed. ENC was the least correlated method ( $\mathrm{r}=0.57$ ), with $31 \%$ of L. lactis genes detected as 


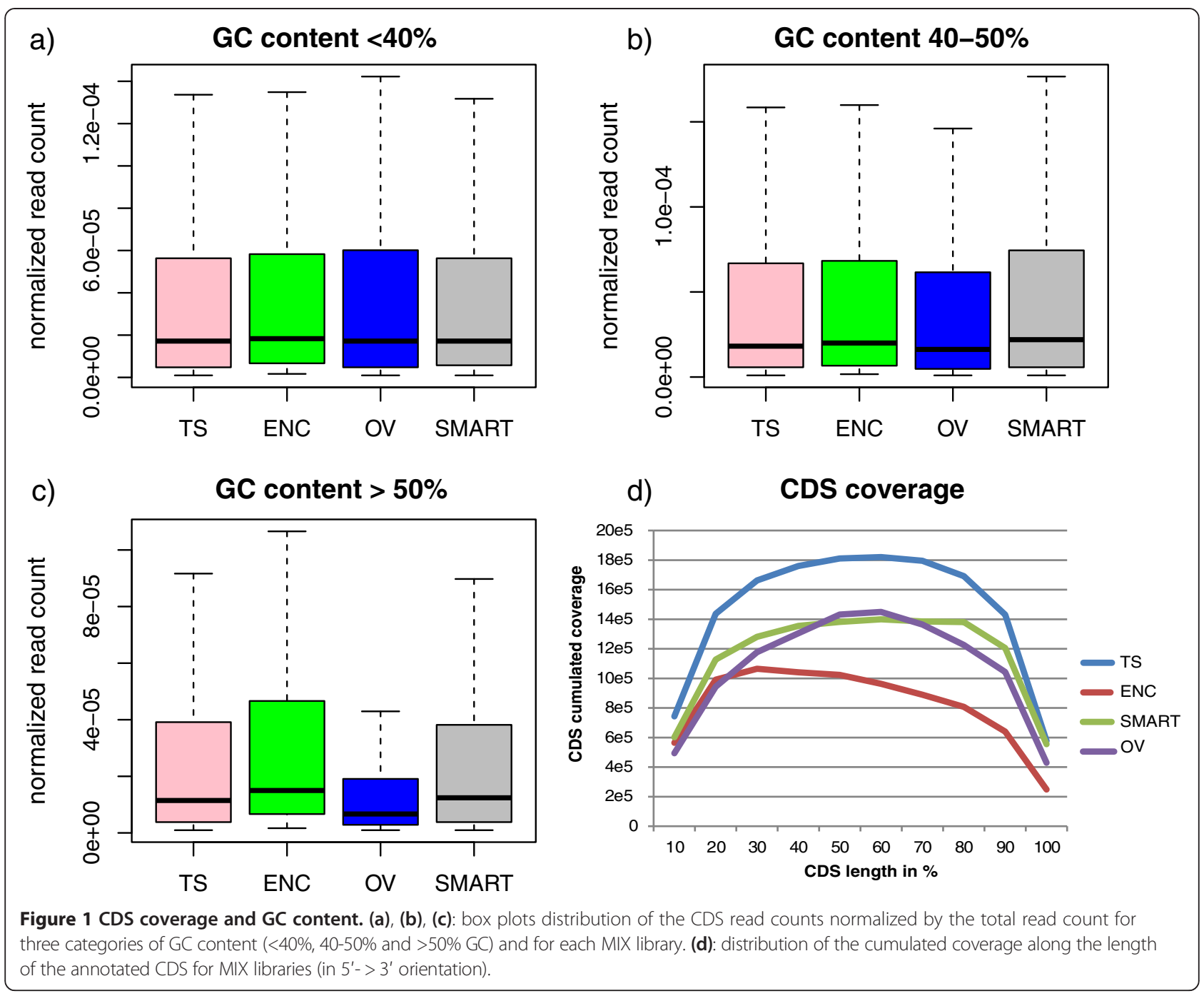

Table 3 Pearson correlation coefficients

\begin{tabular}{|c|c|c|c|c|c|}
\hline & & $\begin{array}{l}\text { L. lactis } \\
\text { vs } \text { MIX }^{\mathrm{a}}\end{array}$ & Replicates $^{\mathrm{b}}$ & $\begin{array}{c}\text { Other } \\
\text { methods } \\
\text { vs TS }\end{array}$ & $\begin{array}{c}\text { Depleted } \\
\text { RNA vs } \\
\text { total RNA }\end{array}$ \\
\hline \multirow[t]{4}{*}{ L. lactis } & TS & 0.992 & 0.886 & & 0.913 \\
\hline & ENC & 0.985 & 0.736 & 0.570 & \\
\hline & OV & 0.974 & 0.995 & 0.696 & 0.971 \\
\hline & SMART & 0.753 & 0.995 & 0.805 & 0.9 \\
\hline \multirow[t]{4}{*}{ MIX } & TS & & 0.958 & & 0.884 \\
\hline & ENC & & 0.980 & 0.498 & \\
\hline & OV & & 0.998 & 0.589 & 0.911 \\
\hline & SMART & & 0.989 & 0.772 & 0.924 \\
\hline
\end{tabular}

Pearson correlation coefficients between:

${ }^{a}$ the $L$. lactis library and the MIX library for each method.

${ }^{b}$ the two replicates for each experiment.

${ }^{\mathrm{C}} \mathrm{ENC}$, OV or SMART L. lactis libraries and TS L. lactis libraries.

${ }^{d}$ the depleted RNA library and the total RNA library for each method. differentially expressed $(\mathrm{p}<0.01)$. The OV experiments performed slightly better $(\mathrm{r}=0.696)$ with $28 \%$ of DEGs. SMART was the best correlated method $(r=0.805)$ and the number of genes detected as differentially expressed in comparison to the TS experiment was not significant. The same comparison made within the MIX samples showed similar results. The SMART MIX sample was the best correlated, even if the percentage of DEGs was slightly higher $(9.2 \%)$ than in the L. lactis libraries (Table 4, Figure 2 and Additional file 2: Figure S6).

We showed previously that the GC content could influence the results. Therefore, we verified if highly differentially expressed genes were correlated with their GC content. This analysis was limited to ENC and OV MIX libraries as the number of genes to be considered in the SMART libraries was not statistically significant. The results showed that the ENC profiles of overexpressed and underexpressed genes were similar (Figure 3). No correlation was evident between the GC content of the genes 
Table 4 Proportions of genes detected as differentially expressed

\begin{tabular}{ccccc}
\hline & $\begin{array}{c}\text { L. lactis } \\
\text { vsL. lactis TS }\end{array}$ & $\begin{array}{c}\text { MIX vs } \\
\text { TS_MIX }\end{array}$ & $\begin{array}{c}\text { L. lactis depleted } \\
\text { RNA vs L. lactis } \\
\text { total RNA }\end{array}$ & $\begin{array}{c}\text { MIX depleted } \\
\text { RNA vs MIX } \\
\text { total RNA }\end{array}$ \\
\hline TS & & & 6.2 & 4.7 \\
ENC & 30.8 & 32.6 & & \\
OV & 27.6 & 35.4 & 8 & 14.5 \\
SMART & 2.2 & 9.2 & 21.3 & 1.1 \\
\hline
\end{tabular}

Proportions of genes detected as differentially expressed between:

${ }^{\mathrm{a} E N C,}$ OV or SMART L. lactis library and TS L. lactis library.

${ }^{\mathrm{b}} \mathrm{ENC}, \mathrm{OV}$ or SMART MIX library and TS MIX library.

${ }^{\mathrm{T}} \mathrm{TS}, \mathrm{OV}$ or SMART L. lactis depleted RNA library and TS,OV or SMART L. lactis total RNA library.

${ }^{\mathrm{d}} \mathrm{TS}, \mathrm{OV}$ or SMART MIX depleted RNA library and TS,OV or SMART MIX total RNA library.

and their differential expression. In contrast, differences in OV gene expression patterns were clearly correlated with the GC content. Specifically, expression of low GC content genes was overestimated and GC-rich genes were penalized, confirming our previous observations that the OV method introduces a bias in favor of low GC genes.

To validate the results of the sequencing experiments, we performed qRT-PCR analysis on certain selected L. lactis genes and compared qRT-PCR quantifications with the read counts of the same genes in the sequenced transcriptomes. A strong positive correlation was observed for the TS and SMART experiments $(r=0.948$ and 0.965 , respectively). ENC showed a slightly lower correlation $(\mathrm{r}=0.899)$, whereas gene expression levels in OV were significantly different $(\mathrm{r}=0.603)$ (Additional file 2: Figure S7). This experiment confirms that, among the "low-input" methods, SMART most reliably represents the mRNA abundance.

\section{Depletion effect}

Ribo-Zero treatment protocol was adapted for SMART and OV experiments to fit with total RNA inputs of $50 \mathrm{ng}$. Hence, we investigated if this technical modification had an impact on the mRNA expression profiles. Linear correlation of gene expression patterns between libraries prepared from total RNA (control libraries) or depleted ones showed similar percentages of mapped reads (Table 2), as well as a strong correlation (Table 3 ) for TS $(r=0.913)$ and OV $(r=0.97)$. No bias in the expression profiles was observed (Table 4 and Additional file 2: Figures S8 and S9).

Curiously, we noticed that in the OV control libraries, the percentage of rRNA reads was significantly lower than expected (less than $78.5 \%$ and $84 \%$ in the L. lactis and MIX libraries, respectively). This observation suggested that the OV RNA priming strategy has a decreased affinity for rRNA. At least two hypotheses could explain this observation: first, the presence of oligo $(\mathrm{dT})$ primers could influence priming of RNA by an unknown mechanism. Second, in this system, random and oligo (dT) primers are coupled to an RNA stretch that is necessary to create the anchoring site for the primer used in subsequent linear amplification. The sequence and length of this RNA stretch, and of the primer, are not available; therefore, we cannot exclude the possibility that they recognize some specific RNA regions, introducing a bias. The same arguments could explain the strong preference of this method towards amplification of low GC content genes.

The comparison between control and depleted libraries prepared using the SMART method revealed a greater bias, especially for the L. lactis sample (Additional file 2: Figures S8 and S9). This probably resulted from the low complexity of the control libraries rather than a bias induced by rRNA depletion. In fact, as the duplication rate was very high in the control libraries (at least 54\%), the number of unique mappable reads and of CDSs detected also strongly decreased (66.6 and 70.9\%). However, Pearson correlation between total and depleted samples was satisfactory (0.900), and the percentage of DEGs was not significant, showing that the expression patterns of the detected genes were not affected.

To verify if the complexity of the library was altered because of an insufficient initial quantity of input RNA, we prepared a library starting from $5 \mathrm{ng}$ of total RNA. As expected, the duplication rate decreased to $14.4 \%$ and read results were comparable to the depleted $L$. lactis library (Additional file 1: Table S4 and Additional file 2: Figure S10). The same experiment from $5 \mathrm{ng}$ total RNA was performed for the MIX and similar results were obtained. Thus, the minimal input for the SMART method should be higher than $1 \mathrm{ng}$ to obtain the best performance.

\section{Other bacterial species}

The same analyses described above were also performed for the other species to confirm the previous conclusions. Our results (Additional file 1: Tables S5, S6 and S7) confirmed the observations made for the L. lactis and MIX samples. Globally, TS and SMART performed best for all the species, whereas OV performances were highly variable. On A. baylyi, OV performed relatively well, based on CDS alignments and Pearson correlation coefficients, whereas in B. subtilis, the results were poor for all measures. Moreover, as in the case of L. lactis, the OV control libraries had lower rRNA read percentages than the SMART and TS libraries. This value varied significantly among species, with $87.6 \%$ of rRNA reads for the E. coli control library and only $54.8 \%$ for B. subtilis control library. Once again, it seems that the OV random priming disfavors rRNA retrotranscription, but at different levels 


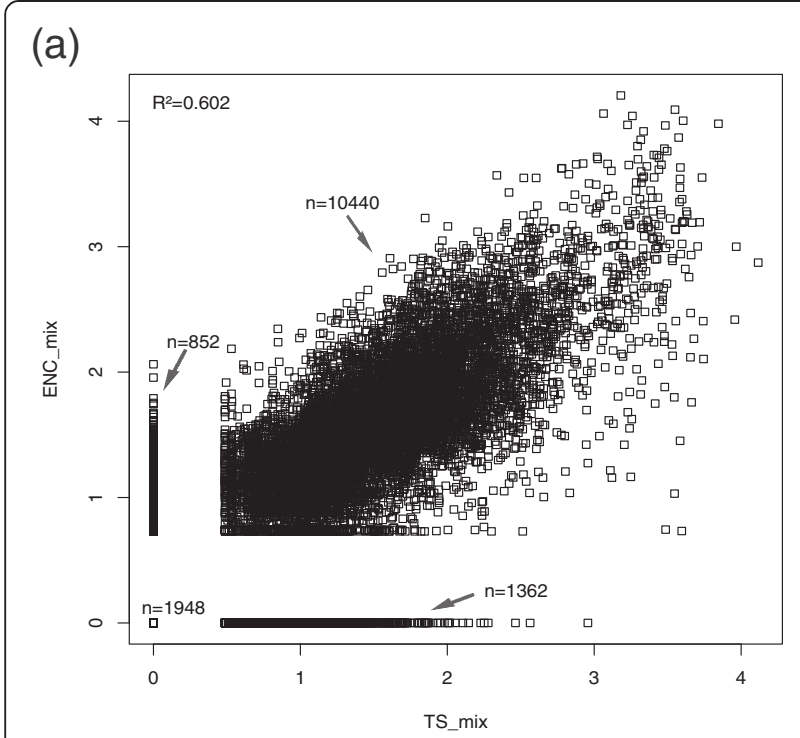

(b)
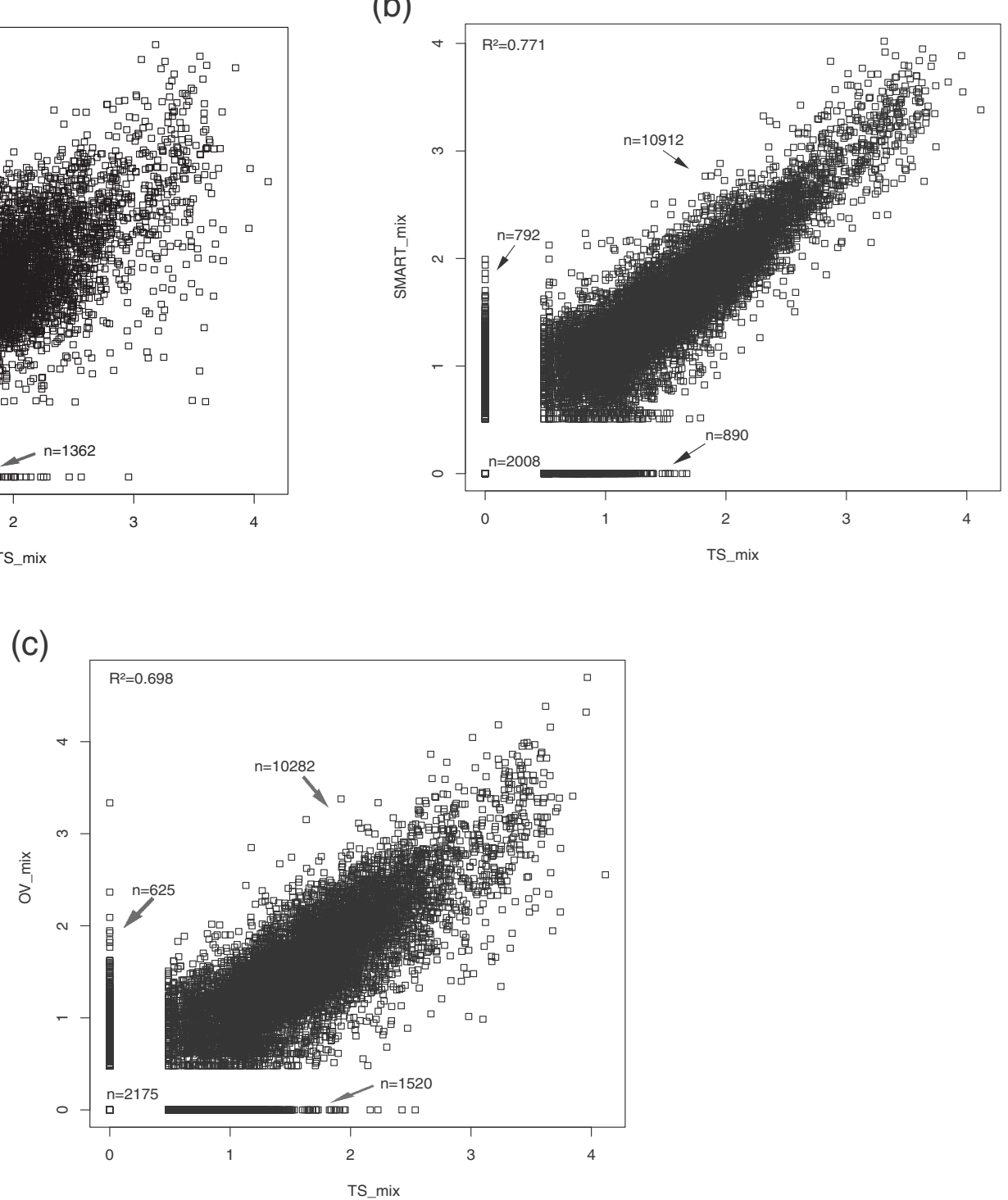

Figure 2 Gene expression profile of TS versus OV, SMART and ENC methods in MIX samples. Gene expression profile from TS_MIX versus (a) ENC_MIX (b) SMART_MIX (c) OV_MIX samples. This figure shows the log scatter plots and the coefficients of determination $\left(R^{2}\right)$ obtained by comparing FPKM values for 14602 annotated CDS in the mix of bacteria.

according to the species. Finally, previous observations of ENC performances were confirmed in the experiments using the other bacterial species: ENC-depleted libraries showed a high percentage of rRNA reads. Similar to L. lactis, the ENC method correlated least well with TS. SMART had the highest correlation coefficient for all the species.

\section{Discussion}

Ribosomal RNA depletion does not introduce significant bias, even at very low inputs

We observed that ribosomal RNA removal treatment by Ribo-Zero is very efficient on every type of sample, as previously shown by Giannoukos et al. [21]. In contrast to this previous study, our protocol was adapted for inputs lower than those defined by the manufacturer to successfully remove rRNA from only $50 \mathrm{ng}$ total RNA. Not only did rRNA reads account for less than $3 \%$, irrespective of the downstream library preparation method, but also the depletion did not seem to introduce biases in mRNA relative abundance, as indicated by the high correlations between total RNA and rRNA-depleted libraries (Pearson coefficient $>0.884$ ).

However, the selective priming strategy adopted by the ENC method seems much less efficient. As selective primers are designed to counter-select rRNA sequences 


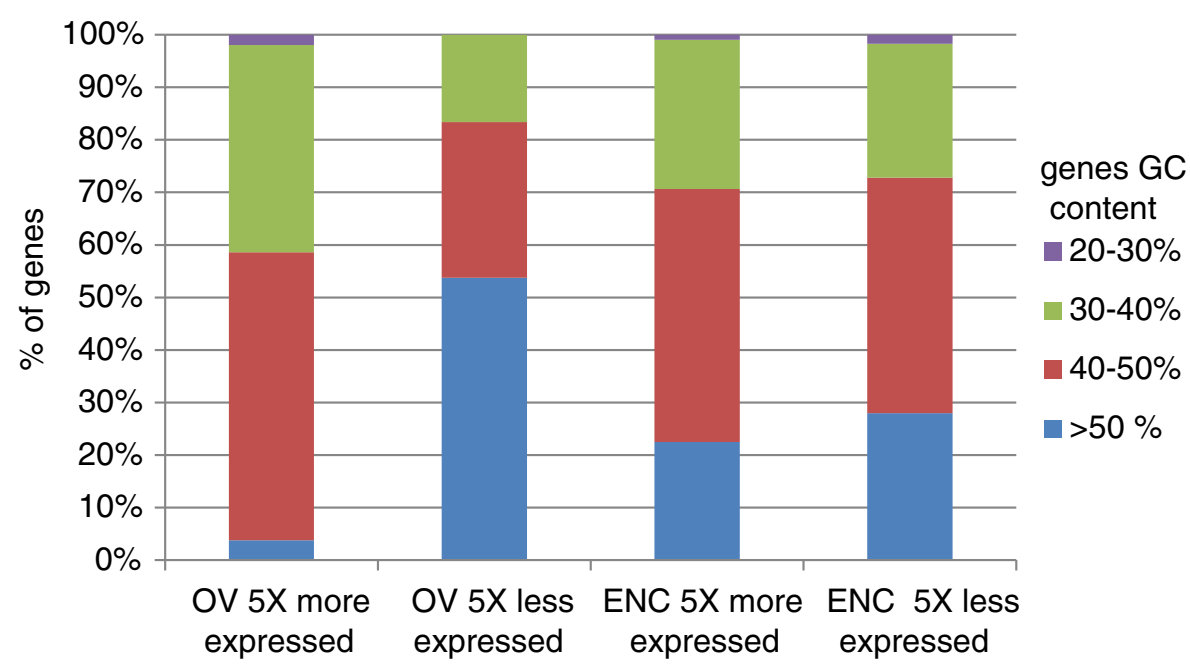

Figure 3 Evaluation of the GC bias in the differential expression analysis. Genes detected as the most differentially expressed were extracted ( 5 fold more or less differentially expressed) for ENC and OV MIX libraries. The proportions of CDS for different GC content intervals were plotted to evaluate the bias introduced by the cDNA synthesis method.

of the most common bacterial species, including our benchmark species, we predict even lower performances when the method is applied to complex environmental samples, which often comprise unknown or poorly characterized organisms.

\section{Technical reproducibility is high}

Technical reproducibility, evaluated by Pearson correlations between replicates, was good for TS, OV and SMART (values >0.886). ENC performed less well for the L. lactis replicates, but not in the MIX, so we are cautious to make conclusions about its robustness. The negligible technical variation within replicates indicated that biases related to each method are also reproducible and are not influenced by the setting up of technical replicates.

\section{TruSeq performs well but is not adapted to "low-input" studies}

The impact of the four library preparation methods on data quality was evaluated by comparing library complexity, specificity in mRNA detection, evenness of CDS coverage in individual and mixed populations, and gene expression patterns. For most of these values, TS performed best, showing the best transcriptional coverage and parameters on CDS alignments (more than 70\%) and gene expression profiles. This is not surprising, as initial experiments were conducted from $4 \mu \mathrm{g}$ total RNA. Lowering the input to $400 \mathrm{ng}$ did not seem to skew the library composition (Additional file 2: Figure S11). Conversely, we tried to construct libraries with only $100 \mathrm{ng}$ total RNA, but the library preparation failed or the yield was very weak (data not shown). Thus, the
TS method seems to be limited by input RNA requirements and does not appear appropriate for most microbial metatranscriptomic studies.

\section{Encore Complete method has a higher cost per sample because of inefficient removal of rRNA}

The ENC library expression profiles were comparable to those obtained with OV and SMART, and they were similar in terms of CDS coverage in both the L. lactis and MIX experiments. However, because of a high \% of rRNA reads, ENC libraries have to be sequenced deeper to obtain sufficient transcriptome coverage. Hence, this method would introduce additional costs to metatranscriptomic studies that require an important sequencing effort to explore the complexity of these samples in depth.

\section{Ovation RNA-Seq system is the best solution at very low inputs but the quantitative information is biased}

The OV library preparation was the simplest and most reliable in terms of library preparation workflow and final yield. It produced several micrograms of doublestranded cDNA starting from less than $0.5 \mathrm{ng}$ RNA (which could be equivalent to about $50 \mathrm{ng}$ if a depletion treatment was necessary). cDNA synthesis was successful even at concentrations of only a few pg (data not shown). A cDNA aliquot could then be used, after shearing, in a standard Illumina library preparation. All the preparation steps are easily amenable to automation.

Reads mapping from OV libraries clearly showed that the CDSs detected were the same found by the other methods and technical reproducibility was satisfactory. By contrast, in the MIX experiments, species distribution was significantly biased. Moreover, we detected evidence of a 
strong bias in gene expression profiles $(\mathrm{r}=0.589)$, particularly in favor of low GC content genes. Another surprising result was the relatively low percentage of rRNA reads in the total RNA control libraries. This led us to hypothesize that the random priming is not truly "random", or that the concurrent presence of oligo(dT) or addition of RNA primer stretches could bias the RNA retrotranscription or cDNA amplification. This should be further explored by including more complex samples with GC and AT-rich species. Finally, this strategy does not provide information about strand orientation, which could be very useful for de novo transcriptome assembly and gene annotation. For all these reasons, we would be very cautious in applying this method for metatranscriptomic studies and, more generally, in quantitative studies.

\section{SMARTer is the best compromise for intermediate RNA inputs}

The SMARTer method is a recent release from Clontech that aims to provide a random and stranded version of the first SMARTer Low Input library kit (oligo(dT) priming), which has been used successfully for eukaryotic single cell transcriptomic studies [26,27]. Indeed, the Low Input oligo(dT) version is robust at very low inputs (even less than 1 ng total RNA) and we experienced this during a marine plankton metatranscriptomic study with satisfactory results (data not shown). The minimal RNA input indicated by the manufacturer for the new random stranded protocol is $1 \mathrm{ng}$ RNA (depleted or total) and, to test it at the minimal input requirements, we prepared control libraries from 1 ng total RNA. Surprisingly, the final library yields were very low and the results obtained from sequencing of these libraries were not satisfactory: high duplication rates coupled to low alignment percentages suggested an insufficient library complexity. The poor efficiency was somewhat surprising but, in opposition to the oligo(dT) low-input method, this protocol does not include an exponential amplification of cDNA before Illumina library preparation. Indeed, single-stranded cDNA is directly amplified with oligonucleotides provided with the kit, which contain Illumina adaptor sequences to obtain a ready-to-sequence library. This could explain why the yields obtained with this method are different to those of the SMARTer low-input version.

The same control experiment performed starting from 5 ng of total RNA produced much better results, leading us to conclude that the minimal RNA input indicated by the manufacturer is not sufficient to preserve sample diversity, even in samples with only one bacterial species. This should be taken into account when choosing the initial RNA quantity to be depleted. Fifty ng seems to be the lower threshold to obtain sufficient library yield and satisfactory transcriptional coverage. In fact, with these inputs, the MIX libraries showed good library complexity, species representation, and gene detection and abundance. However, we could not estimate the exact depleted RNA quantity used for cDNA synthesis after RNA depletion. The rRNA quantity present in a given sample is variable, and depends on growth conditions and the state of the cell at the moment of extraction. It has been demonstrated that non-cultivated environmental bacterial cells contain less mRNA molecules than laboratory strains. For example, only $\sim 200$ transcripts are present simultaneously in a marine bacterial cell versus $\sim 1800$ mRNA molecules in exponentially growing $E$. coli [28]. mRNA yield after depletion could vary and easily be closer to $1 \mathrm{ng}$. Finally, the SMARTer method appears the most satisfying solution for metatranscriptomic studies. However, we propose that more than 50 ng of total RNA should be used to produce robust results.

\section{Gene expression patterns are dependent on the library construction method}

One of the most relevant results of this study is the difference in gene expression patterns among the methods. We clearly demonstrated that comparison of ENC or OV data with TS or SMART would lead to false DEGs detection. By contrast, TS and SMART libraries appear to produce more accurate results, which were corroborated by qRT-PCR experiments. This emphasizes that comparison of RNA-Seq studies performed using different library protocols could be hazardous, and should be carefully evaluated. Knowledge of biases of each method, as provided in the present study, is essential to help interpret the results.

\section{Conclusions}

In this work, we evaluated the impact of cDNA synthesis and library preparation methods on a simplified metatranscriptome. We used four well-characterized bacterial species, individually or pooled together, to mimic a controlled metatranscriptome. First, we showed that the ribosomal depletion treatment by Ribo-Zero efficiently reduces the rRNA reads and does not introduce significant bias, even at an input as low as $50 \mathrm{ng}$ of total RNA. We therefore strongly suggest applying this treatment before library preparation to reduce sequencing costs, particularly in metatranscriptomics studies where deep coverage is needed.

Second, the best library preparation method was closely associated with the initial total RNA quantity used for cDNA synthesis. The TruSeq Stranded protocol produces accurate and reproducible transcriptomic patterns when RNA quantity is not limiting. However, it is not realistic to apply this method for metatranscriptomic samples, usually characterized by very low RNA quantities. Among the methods adapted to lower-input RNA, SMARTer Stranded performed adequately both in terms of library 
yield and data quality. It has the advantage of retaining the coding strand orientation information and it would be suitable for quantitative analysis as shown by the consistency of gene expression patterns. Importantly, we show that TS and SMART results are comparable and can be used together in DEGs analyses. Finally, with very low amounts, the Ovation RNA-Seq System V2 is the only one to guarantee the success of library preparation and sequencing. Nevertheless, this method introduces a significant bias in species abundance and gene expression patterns, probably at priming steps in reverse transcription or linear amplification. Therefore, it may not be helpful for quantitative analysis. However, it allows the production of gene catalogs with extremely low-input samples, which could not be explored otherwise. Thus, the present study provides guidelines that allow the choice of the best-fitted library preparation method for microbial metatranscriptomic studies.

\section{Methods}

\section{Bacterial cultures and lysis}

E. coli MG1655 and B. subtilis 168 were grown in $10 \mathrm{ml}$ LB broth with shaking at $37^{\circ} \mathrm{C}$ to an O.D. ${ }_{600}$ of approximately 0.5 . L. lactis MG1363 was grown in $10 \mathrm{ml} \mathrm{M17}$ broth supplemented with $0.5 \%$ glucose, without shaking, at $30^{\circ} \mathrm{C}$ to an O.D.600 of about 0.5. A. baylyi ADP1 was grown in $10 \mathrm{ml}$ MAS broth at $30^{\circ} \mathrm{C}$ with shaking to an O.D. 600 of approximately 0.7 . For each culture, 6-9-ml aliquots, corresponding to about $1 \times 10^{8}$ cells, were harvested by centrifugation at $6000 \times \mathrm{g}$ for $5 \mathrm{~min}$ at $4^{\circ} \mathrm{C}$. The supernatant was removed. Cell lysis was performed differently depending on $\mathrm{Gram}^{+}$or Gram ${ }^{-}$species. L. lactis and B. subtilis pellets $\left(\mathrm{Gram}^{+}\right)$were resuspended in $100 \mu \mathrm{L}$ TE buffer $(10 \mathrm{mM}$ TrisHCl, $1 \mathrm{mM}$ EDTA, pH 8) plus $10 \mu$ lysozyme $(150 \mathrm{mg} / \mathrm{ml}$, Sigma, St Louis, MO) and $20 \mu \mathrm{l}$ proteinase $\mathrm{K}$ (10 mg/ml, Qiagen, Valencia, CA). Samples were incubated at room temperature for 10 minutes with $10 \mathrm{sec}$ vortexing every 2 minutes during incubation. E. coli and A. baylyi pellets were resuspended in $200 \mu \mathrm{l}$ of preheated $\left(95^{\circ} \mathrm{C}\right)$ Max Bacterial Enhancement Reagent (Ambion, Austin, TX) and the tubes were incubated for 4 minutes at $95^{\circ} \mathrm{C}$.

\section{RNA extractions}

RNA extractions were performed immediately after lysis by using TRIzol solution (Ambion), followed by chloroform phase separation and isopropanol/ethanol RNA precipitation. After a first quantification by Qubit 2.0 fluorometer using Qubit RNA HS Assay Kit (Life Technologies, Carlsbad, CA), $10 \mu \mathrm{g}$ total RNA aliquots were treated with Turbo DNA-free kit (Ambion), according to the manufacturer's instructions. After 30 minutes of incubation and final addition of the inactivation reagent, the supernatant was recovered and the Qubit 2.0 fluorometer was used to quantify the RNA.
Capillary electrophoresis on an Agilent Bioanalyzer (Agilent Technologies, Santa Clara, CA) was used to analyze approximately $3 \mathrm{ng}$ of each RNA, using the RNA 6000 Pico LabChip kit. All RNA samples showed RIN values between 8 and 9.7. Assessment of the efficiency of DNAse treatment by PCR on 10 ng total RNA using strain-specific primers completed the validation of the samples. Phusion High Fidelity PCR Master Mix (Life Technologies) was used to run reactions which included a positive (fresh colony lysate in water) and a negative (nuclease-free water) amplification control. Equimolar amounts of total RNA from the four species were pooled to form the MIX sample. This was the starting material for all the synthetic metatranscriptome experiments.

\section{rRNA removal treatments}

A Ribo-Zero Magnetic Kit for Bacteria (Epicentre, Madison, WI) was used to deplete ribosomal RNA before each cDNA synthesis by TS, SMART or OV methods. The Ribo-Zero depletion protocol was modified to be adapted to different RNA input amounts. Different total RNA inputs were depleted, varying from $50 \mathrm{ng}$ up to $4 \mu \mathrm{g}$. The volume of magnetic beads and rRNA removal solution varied according to the RNA input as follows: $225 \mu \mathrm{l}$ beads and $10 \mu \mathrm{l}$ removal solution in a final volume of $40 \mu \mathrm{l}$ for inputs >1 $\mu$ g; $125 \mu \mathrm{l}$ beads and $4 \mu \mathrm{l}$ rRNA removal solution in a $20 \mu \mathrm{L}$ final reaction volume for $100 \mathrm{ng}$ to $1 \mu \mathrm{g}$ inputs; $90 \mu \mathrm{l}$ beads and $2 \mu \mathrm{l}$ rRNA removal solution in a $20 \mu \mathrm{l}$ reaction volume for $<100 \mathrm{ng}$ inputs. Except for these modifications, depletion was performed according to the manufacturer's instructions.

Depleted RNA was purified with the RNA Clean and Concentrator-5 kit (ZymoResearch, Irvine, CA), following the procedure described for retention of $>17$ nt RNA fragments. RNA was eluted in $8 \mu \mathrm{l}$ nuclease-free water in two elution steps to maximize recovery. When the total RNA input was $>$ or equal to $400 \mathrm{ng}$, a qualitative and quantitative assessment was performed using $1 \mu \mathrm{l}$ of depleted RNA diluted to 1:1 with water. One $\mu \mathrm{l}$ was used for quantification by the Qubit RNA HS Assay and $1 \mu \mathrm{l}$ was run on an Agilent Bioanalyzer, using the RNA 6000 Pico LabChip kit. The Agilent profile showed a dominating small size ( $<200 \mathrm{nt})$ peak presumably corresponding to $5 \mathrm{~S}$ and tRNA species. No $16 \mathrm{~S}$ and $23 \mathrm{~S}$ peaks were detectable. Upon Qubit quantification, recovery from $400 \mathrm{ng}$ inputs was approximately $8 \%$.

\section{Sequencing libraries TruSeq libraries}

Libraries were constructed using depleted RNA obtained from $4 \mu \mathrm{g}, 400 \mathrm{ng}$ and $100 \mathrm{ng}$ total RNA. In the case of $4 \mu \mathrm{g}$ total RNA input in depletion, RNA was quantified by Qubit and $30 \mathrm{ng}$ of depleted RNA was used for 
library preparation. In the other cases, RNA was not quantifiable and the entire volume was used for library preparation. The TruSeq Stranded mRNA Sample Preparation kit (Illumina, San Diego, CA) was used. This kit includes a first poly(A) + RNA selection step by oligo (dT) beads, which may be skipped when working with rRNA-depleted RNAs, as in our case. Therefore, we introduced depleted RNAs in the preparation at the RNA fragmentation and priming step. Five $\mu \mathrm{l}$ of depleted RNA was directly added to $13 \mu \mathrm{l}$ of Fragment, Prime, Finish mix and incubated for $8 \mathrm{~min}$ at $94^{\circ} \mathrm{C}$ for RNA fragmentation and priming. Thereafter, the libraries were prepared according to the manufacturer's protocol without further modifications. Total RNA control libraries were constructed from $50 \mathrm{ng}$ of total RNA following the same protocol modifications (poly $(\mathrm{A})+$ selection step skipped).

\section{Ovation libraries}

Depleted RNA obtained from Ribo-Zero treatment of 50 ng total RNA was used to synthetize and amplify cDNA using the Ovation RNA-Seq System Version 2 (NuGEN, San Carlos, CA), following the manufacturer's protocol. Control cDNA libraries were also prepared from $0.5 \mathrm{ng}$ total RNA following the same protocol. cDNA yield, measured fluorometrically by Qubit DNA HS Assay, was $>2 \mu \mathrm{g}$. An Agilent Bioanalyzer evaluated the cDNA profile using a DNA High Sensitivity LabChip kit. cDNA (500 ng) was sheared by a Covaris E210 instrument (Covaris, Woburn, MA) under the following conditions: $10 \%$ duty cycle, $5 \%$ intensity, 200 cycles per burst, $300 \mathrm{sec}$ in frequency sweeping mode.

Sheared cDNA was used for Illumina library preparation by a semi-automatized protocol. Briefly, end repair, A-tailing and Illumina compatible adaptors (BiooScientific, Austin, TX) ligation were performed using the SPRIWorks Library Preparation System and SPRI TE instrument (Beckmann Coulter Genomics, Danvers, MA), according to the manufacturer's protocol. No size selection was applied, such that most of the fragments were recovered. Twelve PCR cycles using a Platinum Pfx Taq Polymerase Kit (Life Technologies) and Illumina adapter-specific primers amplified the DNA fragments. AMPure XP beads (0.8×; Beckmann Coulter Genomics) were used to purify the libraries.

\section{Encore Complete libraries}

One hundred ng total RNA was used to prepare libraries for the Encore Complete Prokaryotic RNA-Seq System (NuGEN), following the manufacturer's protocol without modifications. Briefly, after first-strand synthesis with selective proprietary primers, and second-strand synthesis with nucleotide analogs, cDNA was sheared by Covaris E210 using the same parameters described for the fragmentation of cDNA obtained using the OV method. Sheared cDNA was ligated to Illumina compatible adaptors containing the nucleotide analog in one strand. Next, the cDNA and adaptor strands containing the analog were selectively removed (Strand Selection), leaving only one cDNA strand with both adaptor sequences attached. This product was then converted into a ready-for-sequencing library by PCR amplification followed by $1 \times$ AMPure XP beads purification.

\section{SMARTer libraries}

Depleted RNA obtained from Ribo-Zero treatment of 50 ng total RNA was used to synthetize and amplify cDNA using the SMARTer Stranded RNA-Seq Kit (Clontech, Mountain View, CA), following the manufacturer's protocol. After first-strand synthesis with SMARTer oligonucleotides, including partial Illumina adaptor sequences, single stranded cDNA was purified by two rounds of clean-up with $1 \times$ AMPure XP beads. The purified product was amplified by 18 cycles PCR with SeqAmp DNA polymerase and the Illumina Index Primer set, both provided in the kit. $1 \times$ AMPure XP beads were used to purify the final libraries. Control libraries were prepared from $1 \mathrm{ng}$ and $5 \mathrm{ng}$ total RNA.

\section{Library quality control and sequencing}

Libraries were quantified by qPCR using the KAPA Library Quantification Kit for Illumina Libraries (KapaBiosystems, Wilmington, MA) and library profiles were assessed using the DNA High Sensitivity LabChip kit on an Agilent Bioanalyzer. Libraries were sequenced on either an Illumina Miseq or a HiSeq2500 instrument using 150 base-length read chemistry in a paired-end mode.

\section{Data quality control pipeline}

After sequencing, an in-house quality control process was applied to reads that passed the Illumina quality filters (raw reads). The sequences of the Illumina adapters and primers used during the library construction were removed from the whole reads. Low-quality nucleotides (quality value $<20$ ) were removed from both ends. The longest sequence without adapters and low-quality bases was kept. Sequences between the second unknown nucleotide $(\mathrm{N})$ and the end of the read were also trimmed. Reads shorter than 30 nucleotides after trimming were discarded. These trimming steps were achieved using internal software based on the FastX package [29]. The reads and their mates that mapped onto run quality control sequences (PhiX genome) were removed. Finally, the reads and their mates that mapped onto a ribosomal sequences database were removed using SortMeRNA software $\mathrm{v} 1.0$ [30]. This software is designed to filter transcriptomic reads data. It contains different rRNA databases and, after treatment, splits the data into two files: 
rRNA reads in one file and no rRNA reads in another. The cleaned reads from the OV and SMART samples were specifically trimmed (respectively the first 12 and three bases in respect of the manufacturer's recommendations). No specific trimming was applied to the cleaned reads from the TS and ENC samples.

All standard metrics (number of reads, number of trimmed bases, number of rRNA reads) were checked on the raw, cleaned and trimmed reads to verify the effects of each treatment. Further analyses were performed on the remaining high-quality data (cleaned reads). Data have been deposited in the European Bioinformatics Institute Short Read Archive (SRA) under accession numbers listed in Additional file 3.

\section{Read mapping onto the reference CDS and statistical analyses} Each sample was mapped against its respective CDS sequences using BWA software version 0.6.1 [31] with its default parameters, allowing two mismatches in the seed (length of 35). The reference sequences were downloaded from the MicroScope website [32,33]. For the pool, the references were put in a unique file. An estimation of the duplicated reads rate was performed on a set of 100000 reads using markDuplicates, which is included in the Picard tools package [34]. Duplicate reads were defined as having both mates aligned at the same position on the genome. The FPKM values were calculated for each sample using the normalized reads counts for each annotated gene $((1000 \times$ read count $) \div$ (number of gene covered bases $\times$ number of mapped fragments in million)). Unmapped reads were removed, retaining only read pairs with both reads aligned to the CDS sequences. The comparison between two different samples was visualized by a scatter plot of the $\mathrm{LOG}_{10}$ of the FPKM. The Pearson correlation coefficient of the gene expressions between the samples were calculated using the $\mathrm{R}$ package version 3.0.2 [35]. The statistical analyses on the read counts were performed with the DESeq package version 1.4.1 [36] to determine the proportion of differentially expressed genes between two samples for a p-value $<0.01$.

\section{qRT-PCR analysis}

qRT-PCR quantification was performed on $10 \mathrm{~L}$. lactis genes. Six of them were selected on the basis of read count levels in the TS experiments, from high (eno and $t u f$ ) and intermediate ( $\mathrm{r} r \mathrm{dE}$ and $\sec A$ ) to low ( $g l t B$ and thiD). The other four genes, tpx, gpmA, llmg0195 (putative NADH dehydrogenase) and llmg2023 (universal stress protein A), were selected among the DEGs observed in the SMART, OV and ENC experiments. Primers designed using the Primer3Plus software are listed in Additional file 1: Table S8. Reverse transcription reactions were carried out on $150 \mathrm{ng}$ L. lactis total RNA using random hexamers and Superscript II RT (Life Technologies), according to the manufacturer's instructions. The synthesized cDNA was used as the template for $\mathrm{qPCR}$ reactions performed using the KAPA SYBR Fast qPCR kit (KapaBiosystems) on an Mx3005P qPCR System (Agilent Technologies). cDNA copies were quantified for each gene relative to a standard curve made from serial dilutions of purified L. lactis genomic DNA. Comparison of RNA-Seq and qRT-PCR quantifications was visualized by a scatter plot of the $\mathrm{LOG}_{10}$ of the read counts and the $\mathrm{LOG}_{10}$ of the qPCR quantification [37].

\section{Availability of supporting data}

The data sets supporting the results of this article are included within this article and its additional files.

\section{Additional files}

Additional file 1: Table S1. Characteristics of each bacterium genome Table S2: Proportions of detected genes and of covered intergenic bases. Table S3. Taxonomic assignation of the MIX libraries reads. Table S4: Sequences statistics for SMART control libraries prepared with 5 ng total RNA. Table S5. Sequences statistics for A. baylyi, B. subtilis, and E. coli libraries. Table S6. Proportions of detected genes and of CDS covered bases for other bacteria species libraries. Table S7. Pearson correlation coefficients related to A. baylyi, B.subtilis, and E. coli libraries comparisons. Table S8. Oligonucleotides used for qRT-PCR analysis.

Additional file 2: Figure S1. Gene expression profile of L. lactis samples versus MIX samples. Figure S2. Gene expression profile of the two replicates from L. lactis samples. Figure $\mathbf{S 3}$. Gene expression profile of the two replicates from MIX samples. Figure S4. Gene expression profile of TS versus OV, SMART and ENC methods in L. lactis samples. Figure S5. mRNA differential expression profile in L. lactis samples. Figure S6. mRNA differential expression profile in MIX samples. Figure S7. Scatter plots of gene expression levels detected by RNA-Seq and qRT-PCR for $10 \mathrm{~L}$. lactis genes. Figure S8. Gene expression profile of depleted RNA L. lactis samples versus total RNA L. lactis samples. Figure S9. Gene expression profile of depleted RNA MIX samples versus total RNA MIX samples. Figure S10: Gene expression profile of SMART control libraries. Figure S11: Comparison between L. lactis TS libraries prepared with two different RNA inputs.

Additional file 3: SRA accession numbers.

\section{Competing interests}

The authors declare that they have no competing interests.

\section{Authors' contributions}

$\mathrm{AA}$ and $\mathrm{CB}$ conceived the project and designed the experiments with contributions from SE, PW, CC, LBr and LG. LBe, CO and AA performed experiments. CB, SE and CDS collected and analyzed the results. CF developed the data quality control pipeline. $A A, C B$ and PW wrote the manuscript with inputs from JMA and CDS. All authors read and approved the final manuscript

\section{Acknowledgements}

We thank Pascale Serror for providing B. subtilis and L. lactis strains as well as for suggestions for culture conditions, Robert Olaso and Emilie Pateau for help in GRT-PCR experiments, Karine Labadie for sequencing workflow support and Andrew Tolonen for useful comments on the manuscript. Funding was provided by France Génomique (ANR-10-INBS-0009-08) as part of Investissements d'Avenir Program and Prometheus (ANR-09-PCS-GENM-217). 


\section{Author details}

'CEA-Institut de Génomique, Genoscope, Centre National de Séquençage, 2 rue Gaston Crémieux, CP5706, F-91057 Evry Cedex, France. 'Université d'Evry, UMR 8030, CP5706 Evry, France. ${ }^{3}$ Centre National de la Recherche Scientifique (CNRS), UMR 8030, CP5706 Evry, France.

Received: 22 May 2014 Accepted: 13 October 2014

Published: 20 October 2014

\section{References}

1. Gilbert JA, Field D, Huang Y, Edwards R, Li W, Gilna P, Joint I: Detection of large numbers of novel sequences in the metatranscriptomes of complex marine microbial communities. PLoS One 2008, 3(8):e3042.

2. Frias-Lopez J, Shi Y, Tyson GW, Coleman ML, Schuster SC, Chisholm SW, Delong EF: Microbial community gene expression in ocean surface waters. Proc Natl Acad Sci U S A 2008, 105(10):3805-3810.

3. Poretsky RS, Hewson I, Sun S, Allen AE, Zehr JP, Moran MA: Comparative day/night metatranscriptomic analysis of microbial communities in the North Pacific subtropical gyre. Environ Microbiol 2009, 11(6):1358-1375.

4. Hewson I, Poretsky RS, Beinart RA, White AE, Shi T, Bench SR, Moisander PH, Paerl RW, Tripp HJ, Montoya JP, Moran MA, Zehr JP: In situ transcriptomic analysis of the globally important keystone N2-fixing taxon Crocosphaera watsonii. ISME J 2009, 3(5):618-631.

5. Hewson I, Poretsky RS, Dyhrman ST, Zielinski B, White AE, Tripp HJ, Montoya JP, Zehr JP: Microbial community gene expression within colonies of the diazotroph, Trichodesmium, from the Southwest Pacific Ocean. ISME J 2009, 3(11):1286-1300

6. Gifford SM, Sharma S, Rinta-Kanto JM, Moran MA: Quantitative analysis of a deeply sequenced marine microbial metatranscriptome. ISME J 2011, 5(3):461-472.

7. Gifford SM, Sharma S, Booth M, Moran MA: Expression patterns reveal niche diversification in a marine microbial assemblage. ISME J 2013 , 7(2):281-298.

8. Leininger S, Urich T, Schloter M, Schwark L, Qi J, Nicol GW, Prosser Jl, Schuster SC, Schleper C: Archaea predominate among ammonia-oxidizing prokaryotes in soils. Nature 2006, 442(7104):806-809.

9. Tveit $A$, Schwacke $R$, Svenning MM, Urich T: Organic carbon transformations in high-Arctic peat soils: key functions and microorganisms. ISME J 2013, 7(2):299-311.

10. Urich T, Lanzen A, Qi J, Huson DH, Schleper C, Schuster SC: Simultaneous assessment of soil microbial community structure and function through analysis of the meta-transcriptome. PLoS One 2008, 3(6):e2527.

11. Gosalbes MJ, Durban A, Pignatelli M, Abellan JJ, Jimenez-Hernandez N, Perez-Cobas AE, Latorre A, Moya A: Metatranscriptomic approach to analyze the functional human gut microbiota. PLoS One 2011, 6(3):e17447.

12. Stewart FJ: Preparation of microbial community cDNA for metatranscriptomic analysis in marine plankton. Methods Enzymol 2013, 531:187-218.

13. Carvalhais LC, Schenk PM: Sample processing and cDNA preparation for microbial metatranscriptomics in complex soil communities. Methods Enzymol 2013, 531:251-267.

14. Neidhardt FC, Umbarger HE: Chemical composition of Escherichia coli. In Escherichia coli and Salmonella: Cellular and Molecular Biology, Volume 1. 2nd edition. Edited by Neidhardt FC. Washington D.C: ASM Press; 1996:13-17.

15. McGrath KC, Thomas-Hall SR, Cheng CT, Leo L, Alexa A, Schmidt S, Schenk PM: Isolation and analysis of mRNA from environmental microbial communities. J Microbiol Methods 2008, 75(2):172-176.

16. Stewart FJ, Ottesen EA, DeLong EF: Development and quantitative analyses of a universal rRNA-subtraction protocol for microbial metatranscriptomics. ISME J 2010, 4(7):896-907.

17. Yi H, Cho YJ, Won S, Lee JE, Jin Yu H, Kim S, Schroth GP, Luo S, Chun J: Duplex-specific nuclease efficiently removes rRNA for prokaryotic RNA-seq. Nucleic Acids Res 2011, 39(20):e140.

18. Peano C, Pietrelli A, Consolandi C, Rossi E, Petiti L, Tagliabue L, De Bellis G, Landini P: An efficient rRNA removal method for RNA sequencing in GC-rich bacteria. Microb Inform Exp 2013, 3(1):1.

19. He S, Wurtzel O, Singh K, Froula JL, Yilmaz S, Tringe SG, Wang Z, Chen F, Lindquist EA, Sorek R, Hugenholtz P: Validation of two ribosomal RNA removal methods for microbial metatranscriptomics. Nat Methods 2010, 7(10):807-812

20. Mettel C, Kim Y, Shrestha PM, Liesack W: Extraction of mRNA from soil. Appl Environ Microbiol 2010, 76(17):5995-6000
21. Giannoukos G, Ciulla DM, Huang K, Haas BJ, Izard J, Levin JZ, Livny J, Earl AM, Gevers D, Ward DV, Nusbaum C, Birren BW, Gnirke A: Efficient and robust RNA-seq process for cultured bacteria and complex community transcriptomes. Genome Biol 2012, 13(3):R23.

22. Adiconis X, Borges-Rivera D, Satija R, Deluca DS, Busby MA, Berlin AM, Sivachenko A, Thompson DA, Wysoker A, Fennell T, Gnirke A, Pochet N, Regev A, Levin JZ: Comparative analysis of RNA sequencing methods for degraded or low-input samples. Nat Methods 2013, 10(7):623-629.

23. Levin JZ, Yassour M, Adiconis X, Nusbaum C, Thompson DA, Friedman N, Gnirke A, Regev A: Comprehensive comparative analysis of strand-specific RNA sequencing methods. Nat Methods 2010, 7(9):709-715.

24. Sun Z, Asmann YW, Nair A, Zhang Y, Wang L, Kalari KR, Bhagwate AV, Baker TR, Carr JM, Kocher JP, Perez EA, Thompson EA: Impact of library preparation on downstream analysis and interpretation of RNA-Seq data: comparison between Illumina PolyA and NuGEN Ovation protocol. PLoS One 2013, 8(8):e71745

25. Malboeuf CM, Yang X, Charlebois P, Qu J, Berlin AM, Casali M, Pesko KN, Boutwell CL, DeVincenzo JP, Ebel GD, Allen TM, Zody MC, Henn MR, Levin JZ: Complete viral RNA genome sequencing of ultra-low copy samples by sequence-independent amplification. Nucleic Acids Res 2013, 41(1):e13.

26. Ramskold D, Luo S, Wang YC, Li R, Deng Q, Faridani OR, Daniels GA Khrebtukova I, Loring JF, Laurent LC, Schroth GP, Sandberg R: Full-length mRNA-Seq from single-cell levels of RNA and individual circulating tumor cells. Nat Biotechnol 2012, 30(8):777-782.

27. Deng Q, Ramskold D, Reinius B, Sandberg R: Single-cell RNA-seq reveals dynamic, random monoallelic gene expression in mammalian cells. Science 2014, 343(6167):193-196.

28. Moran MA, Satinsky B, Gifford SM, Luo H, Rivers A, Chan LK, Meng J, Durham BP, Shen C, Varaljay VA, Smith CB, Yager PL, Hopkinson BM: Sizing up metatranscriptomics. ISME J 2013, 7(2):237-243.

29. FASTX-Toolkit. [http://hannonlab.cshl.edu/fastx_toolkit/index.html]

30. Kopylova E, Noe L, Touzet H: SortMeRNA: fast and accurate filtering of ribosomal RNAs in metatranscriptomic data. Bioinformatics 2012 28(24):3211-3217.

31. Li H, Durbin R: Fast and accurate short read alignment with Burrows-Wheeler transform. Bioinformatics 2009, 25(14):1754-1760.

32. Vallenet D, Belda E, Calteau A, Cruveiller S, Engelen S, Lajus A, Le Fevre F, Longin C, Mornico D, Roche D, Rouy Z, Salvignol G, Scarpelli C, Thil Smith AA, Weiman M, Medigue C: MicroScope-an integrated microbial resource for the curation and comparative analysis of genomic and metabolic data. Nucleic Acids Res 2013, 41(Database issue):D636-D647.

33. MicroScope. Microbial Genome Annotation \& Analysis Platform. In [http://www.cns.fr/agc/microscope/home/index.php]

34. Picard. In [http://broadinstitute.github.io/picard]

35. The R Project for Statistical Computing. In [http://www.r-project.org/]

36. Anders $\mathrm{S}$, Huber W: Differential expression analysis for sequence count data. Genome Biol 2010, 11(10):R106.

37. Asmann YW, Klee EW, Thompson EA, Perez EA, Middha S, Oberg AL, Therneau TM, Smith DI, Poland GA, Wieben ED, Kocher JP: 3' tag digital gene expression profiling of human brain and universal reference RNA using Illumina Genome Analyzer. BMC Genomics 2009, 10:531.

doi:10.1186/1471-2164-15-912

Cite this article as: Alberti et al:: Comparison of library preparation methods reveals their impact on interpretation of metatranscriptomic data. BMC Genomics 2014 15:912 